\title{
De « l'ombre légère » à la « machine à écrire
} familiale »

L'écriture quotidienne des femmes

Isabelle Lacoue-Labarthe et Sylvie Mouysset

\section{(2) OpenEdition}

\section{Journals}

Édition électronique

URL : http://journals.openedition.org/clio/10489

DOI : $10.4000 /$ clio. 10489

ISSN : 1777-5299

Éditeur

Belin

Édition imprimée

Date de publication : 1 mai 2012

Pagination : 7-20

ISSN : 1252-7017

Référence électronique

Isabelle Lacoue-Labarthe et Sylvie Mouysset, «De « l'ombre légère » à la « machine à écrire familiale » », Clio. Femmes, Genre, Histoire [En ligne], 35| 2012, mis en ligne le 01 mai 2014, consulté le 21

septembre 2020. URL : http://journals.openedition.org/clio/10489; DOI : https://doi.org/10.4000/clio. 10489

Tous droits réservés 


\title{
De « l'ombre légère " à la « machine à écrire familiale » : l'écriture quotidienne des femmes
}

\author{
Isabelle LACOUE-LABARTHE \& Sylvie MOUYSSET
}

Les écritures du quotidien sont aujourd'hui bien souvent affaire de femmes ${ }^{1}$; celles-ci recueillent, sauvent et agencent l'éphémère patrimoine mémoriel qui, sans le secours de quelque sœur, tante ou vieille cousine, serait voué à l'oubli. Véritables «machines à écrire familiales", selon la formule chère à Bernard Lahire, les femmes notent, archivent et mettent en forme les traces d'un passé ordinaire qui prend tout son sens à la faveur de leurs commentaires et gardent la famille en vie ${ }^{2}$. Avant le XIXe siècle, la mémoire familiale n'était pas dans les mains des femmes; ou alors le fait semblait exceptionnel. Jadis ombres légères, elles ont, en prenant la plume, changé de place, comme le soulignait Daniel Fabre en 1997 :

Alors que l'écrit domestique central était le carnet où s'égrenait une sorte de livre de raison (l'agenda du berger, du vigneron ou du maraîcher), donc une affaire d'homme, voici maintenant les femmes en charge de la comptabilité et de tous les papiers afférents à l'exploitation, assumant une vraie tâche de secrétariat d'entreprise, alors que, par ailleurs, elles se révèlent bien plus grandes lectrices ${ }^{3}$.

D'exceptionnelle, éphémère et interstitielle, la pratique d'écriture est peu à peu devenue régulière et même quasi exclusivement féminine. Ses formes sont des plus variées, du simple post-it laissé aujourd'hui sur la porte d'un réfrigérateur à la longue lettre envoyée autrefois par une fille à sa mère. Pour les qualifier, les historiens français affectionnent la formule "écrits du for privé », inventée par

\footnotetext{
Siblot 2006.

Lahire 1993 : 153 et 1997 ; Albert 1993 ; Fine, Labro \& Lorquin 1993.

Fabre $1997: 16$.
} 
Madeleine Foisil en 1986 dans un chapitre devenu classique du troisième volume de l'Histoire de la vie privée. Forgée à partir du concept religieux de "for intérieur", cette formule qui ne va pas de soi désigne un ensemble varié de papiers personnels, d'écritures de soi ou d'ego-documents ${ }^{4}$. On trouve alors ici les livres de raison ou de comptes, les livres de famille, les diaires et éphémérides, les mémoires, autobiographies et journaux de toute nature (personnel ou « intime", de voyage, de campagne, de prison...), la correspondance bien sûr aussi et, d'une manière générale, tous les textes produits hors institution et témoignant d'une prise de parole personnelle d'un individu sur lui-même, les siens, sa communauté, le monde tel qu'il le vit, l'imagine et le perçoit, à travers son regard et sa plume singulière.

Longtemps, ces écrits multiformes du quotidien sont restés invisibles et muets, dans le secret de nos greniers. Ils sont aujourd'hui l'objet de notre attention, et tout particulièrement ceux, moins nombreux et plus silencieux encore, qui se font l'écho de voix féminines.

\section{D'une longue absence à une réelle visibilité}

Le silence des femmes... Et s'il en était autrement? Si l'on n'avait pas, jusqu'à une époque récente, suffisamment écouté les voix féminines, pourtant si sonores et polyphoniques dans leurs manières de dire leur univers quotidien? On a souvent avancé l'absence de sources, ainsi que le regrette Gabrielle Houbre dans l'éditorial du volume de Clio consacré au «temps des jeunes filles » :

$\mathrm{Au}$ terme de ce numéro subsiste, faute de sources directes, l'insatisfaction de n'avoir pu mieux approcher l'intimité de ces jeunes filles, figures évanescentes de l'histoire et dont l'extrême discrétion, sauf à rencontrer la tendre persévérance d'un Philippe Lejeune à traquer le Moi des demoiselles, reste de mise jusqu'au XX ${ }^{\mathrm{e}}$ siècle ${ }^{5}$.

Aujourd'hui, au vu d'une florissante activité éditoriale, il semble que lorsqu'on cherche les écrits féminins, on les trouve! Cette «mémoire restituée » (M. Caffiero) est l'œuvre de chercheur-e-s qui reconstituent patiemment le fil ténu d'une histoire longtemps illisible. Le sujet est

4 Le terme «egodocument» est attribué à l'historien hollandais Jacob Presser (1899-1970) ; voir à ce propos Dekker 2002.

5 Houbre 1996. 
immense, même si l'on réduit la voilure à la seule culture écrite du quotidien. Celle-ci a profité, depuis près d'un demi-siècle, d'un renouvellement historiographique vivifiant, motivé par le dynamisme de l'histoire sociale et des mentalités, ainsi que de la micro-histoire. En 1961, Robert Mandrou invitait à la (re)découverte de textes émanant de gens ordinaires, écrivains sans qualité, modestes témoins de la banalité de leur existencé ${ }^{6}$. De Gouberville à Ménétra, de belles éditions ont vu le jour. En 2003 est né un groupe de recherche sur "les écrits du for privé en France de la fin du Moyen Âge à 1914 », dirigé par Jean-Pierre Bardet et François-Joseph Ruggiu, qui s'est fixé deux objectifs: la diffusion des savoirs par l'organisation de rencontres et l'édition de travaux individuels ou collectifs, et la construction d'une base de données des écrits ordinaires dans laquelle on a pris soin d'insérer une rubrique « genre » ${ }^{7}$.

L'écriture des femmes n'a pas toujours été absente des préoccupations des chercheur-e-s, mais elle a mis du temps à s'imposer comme objet légitime ; stigmatisée comme l'épanchement de femmes «bavardes et écrivassières » par certaines femmes ellesmêmes - et non des moindres ${ }^{8}-$, elle a parfois été naturalisée, ravalée à un simple discours d'opprimées. L'existence d'une «écriture féminine » a ainsi été débattue à partir de la fin des années $1970^{\circ}$; la découverte de l'abondance des journaux, correspondances et récits intimes émanant de plumes féminines a contribué à une réflexion parfois éloignée de toute préoccupation historique sans lancer d'étude systématique de ces écrits. Pour ces derniers, la lumière est souvent venue des littéraires.

Les journaux de jeunes filles et de jeunes femmes qui fleurissent dès la fin du XVIII siècle ont été au cœur des recherches menées par le sociolinguiste Philippe Lejeune. À l'aube des années 1970, celui-ci a révélé que, si l'on publie « surtout des journaux d'hommes $(80 \%)$ », ce sont «majoritairement les femmes qui écrivent des journaux

6 Mandrou 1961.

7 Groupe de recherche CNRS n²649 sur «Les écrits du for privé en France du Moyen Âge à nos jours", dirigé par Jean-Pierre Bardet \& François-Joseph Ruggiu : www.ecritsduforprive.fr.

8 Beauvoir 1976 [1949] : 628.

9 Stistrup Jensen 2000. 
$(65 \%) »^{10}$. Pionnier de l'analyse autobiographique ${ }^{11}$, il est cofondateur de l'Association pour l'autobiographie et le Patrimoine Autobiographique. Celle-ci accueille toutes sortes de documents autobiographiques inédits, les recense dans une publication annuelle, le Garde-mémoire, dont les derniers numéros confirment la primauté des écrits féminins ${ }^{12}$. L'anthropologue Anna Iuso dresse pareil constat à propos du fonds toscan de l'Archivio Diaristico Nazionale, qui accueille, depuis 1984, mémoires, correspondances, journaux et offre un « prix de la meilleure autobiographie de l'année $»^{13}$.

Aujourd'hui encore, bien des études consacrées aux écrits féminins émanent de chercheur-e-s littéraires. Explorant ceux de Marceline Desbordes-Valmore ${ }^{14}$, de George Sand ${ }^{15}$ mais aussi d'Eugénie de Guérin ${ }^{16}$, Christine Planté interroge ainsi le genre de l'écriture épistolaire depuis la fin du XVII ${ }^{\mathrm{e}}$ siècle ${ }^{17}$.

Les lettres de Madame de Sévigné ont longuement accaparé l'œil bienveillant de Roger Duchêne ${ }^{18}$. Catherine Viollet et l'équipe "Genèse et Autobiographie » de l'Institut des Textes et Manuscrits Modernes (ITEM) font des écrits autobiographiques et épistolaires féminins un point central de leurs recherches, à l'instar de Françoise Simonet-Tenant qui, au-delà de travaux consacrés à Catherine Pozzi ${ }^{19}$, aborde toutes les formes de l'écriture quotidienne au féminin.

Lejeune 2005 : 96.

1 Cf. entre autres Lejeune 1993a et 1993b.

12 Nous remercions vivement Philippe Lejeune pour ses renseignements précieux. Site de l'APA : http://sitapa.free.fr. L'APA française a, depuis 2002, une petite sœur belge, l'APA-Bel.

13 Iuso 1997. Voir http://www.archiviodiari.it/default.htm. Le lectorat de ce site est féminin : Iuso 1998 .

14 Planté 1992.

15 Planté 1998b.

16 Planté 1994.

17 Planté 1998a : parmi les auteur-e-s, on peut lire les historiennes Danièle Poublan, Cécile Dauphin et Michèle Riot-Sarcey. Voir aussi Fabre 2000.

18 Duchêne 1970.

19 Simonet-Tenant 2001. 
Dans l'engouement pour ce type de documents, les anthropologues ne sont pas en reste ${ }^{20}$, ni même les historien-ne-s. Linda Timmermans déplorait, en 1993, le piètre nombre de travaux sur les mémorialistes féminines ${ }^{21}$. Les recherches d'Henri Rossi sur les mémoires aristocratiques féminins, ou celles d'Isabelle Poutrin et de Laura Verciani sur les autobiographies spirituelles, sont venues, depuis, combler en partie cette lacune ${ }^{22}$. Michelle Perrot a très tôt vu dans ces écrits des sources de premier ordre ${ }^{23}$ et mis en lumière les lettres des filles de Karl Marx ${ }^{24}$, le journal de Caroline Brame ${ }^{25}$, retrouvé par Georges Ribeill aux puces de Saint-Ouen, et d'autres textes de jeunes filles se penchant «au miroir de l'âme ${ }^{26}$, traces rescapées des pudeurs féminines ou du désintérêt des héritiers. De son côté, Marie-Claire Hoock-Demarle étudie l'intense activité des épistolières européennes, en particulier allemandes, de la fin du XVIII à l'aube du XX $\mathrm{XX}^{\mathrm{e}}$ siècle $^{27}$.

Cette dynamique est perceptible dans l'ensemble de l'Europe ; Marina Caffiero en témoigne ici pour l'Italie où les travaux en nombre disent l'abondance des écrits quotidiens de religieuses mais aussi de laïques, textes spontanés ou commandés, à vocation privée et parfois publique ${ }^{28}$. Cette richesse apparaît aussi dans l'ambitieuse collection américaine lancée en 1996 par Margaret King et Albert Rabil, «The Other Voice in Early Modern Europe » (University of Chicago Press) ; parmi la soixantaine de textes féminins publiés à ce jour - correspondances et Mémoires pour près de la moitié d'entre eux -, beaucoup ont été écrits par des Italiennes. Depuis les années 1980, groupes de recherches et initiatives éditoriales (dictionnaires et répertoires biographiques, par exemple) consacrés aux écrits ordinaires de femmes, célèbres ou pas, originaires de la Catalogne,

20 Cf. Iuso dans ce numéro, et aussi 1997, 1998 ; Fine 2000 ; Fine, Labro \& Lorquin 1993.

21 Timmermans 1993.

22 Poutrin 1995, 2005 et Verciani 2001.

23 Perrot 1987.

4 Perrot 1979.

5 Perrot \& Ribeill 1985.

Perrot 1986.

Hoock-Demarle 1990 et 2008.

28 Cf. Caffiero dans ce numéro; Caffiero \& Venzo 2007. 
de Valence ou des Îles Baléares se sont multipliés, nous disent Eulàlia Miralles et Verònica Zaragoza, en particulier pour le Moyen Âge et la Renaissance ${ }^{29}$. Certains travaux récents, aussi vivifiants qu'encourageants, traduisent effervescence et vigueur d'un mouvement qui dépasse désormais largement le cadre européen ${ }^{30}$.

\section{Cent fois sur le métier remettons notre ouvrage}

Le chantier est immense et l'ouvrage devra maintes fois être remis sur le métier. Nous souhaitons seulement ici tendre quelques fils de trame et contribuer à faire fructifier la réflexion commune.

Nous avons fait le choix de la diversité des contextes et des espaces d'écriture ${ }^{31}$. De fait, la sociologie des femmes écrivaines de leur vie minuscule est d'autant plus complexe à cerner précisément ici : qu'y a-t-il, en effet, de commun entre les épistolières florentines $\mathrm{du} \mathrm{XV}^{\mathrm{e}}$ siècle et les "pionnières » sionistes du XXe siècle ? À première vue, pas grand chose. Mais si l'on y regarde de plus près, des points de convergence apparaissent et permettent un premier inventaire des pistes à poursuivre. Seules les femmes lettrées sont capables de tenir une plume, même s'il n'est pas nécessaire de maitriser la graphie pour décider de coucher les détails de son existence: on peut écrire «morogie » pour hémorragie, «marmelade d'aprico » (I. Luciani), ou «Trusques » pour Étrusques (A. Iuso).

Ainsi une histoire au ras du sol devrait-elle sans aucun doute révéler les trésors insoupçonnés de fonds jusqu'ici inexplorés : le journal d'Alia Rachmanova ou celui d'Evguenia Kisseliova sont quelques exemples de récentes découvertes ${ }^{32}$.

29 On se reportera à l'abondante bibliographie fournie par les deux historiennes dans ce numéro.

30 Le dernier colloque du GdR n 2649 (juillet 2011), portait sur «Les usages de l'écrit du for privé (Afriques, Amériques, Asies, Occidents, Orients)». L’édition des actes, prévue en 2012, proposera un utile bilan actualisé des recherches extra-européennes.

31 Le présent volume aborde principalement les sociétés européennes. Des recherches innovantes concernent cependant d'autres aires, telle l'Afrique (Barthélémy 2009, Mbodj-Pouye \& Ficquet 2009).

32 Rachmanova 2010 et Kisseliova 2000. 
Nous ouvrons ce recueil avec l'article d'Isabelle Luciani sur les écrits du for privé féminins provençaux. Une trentaine de femmes, trois fois moins que d'hommes, ont tenu leur livre de raison. Ce petit tiers pose déjà une question essentielle, celle du nombre de scriptrices. Combien étaient-elles, quelle est la part du féminin dans l'écriture du quotidien? Jetons un coup d'œil sur la base de données des écrits du for privé français $^{33}$ : pour 1865 auteurs recensés à ce jour (et 1820 manuscrits), on compte 197 femmes, 1585 hommes et 83 de genre inconnu. Seulement un peu plus de $10 \%$ des auteurs sont donc des femmes. Le cas provençal suggère cependant que cette proportion pourrait être supérieure à celle jusqu'ici repérée au plan national, avant même la fin du XVIII ${ }^{\mathrm{e}}$ siècle. Dans cette première époque de l'écrit féminin, du XVI au premier XVIII ${ }^{\mathrm{e}}$ siècle, peut-on continuer à penser qu'à l'aune de leurs écrits, les femmes sont «ombre légère »? C'est à voir. Un fait est certain, en revanche, c'est le nombre impressionnant d'écrits féminins à découvrir de l'aube des Lumières à nos jours (M.-C. Hoock-Demarle, D. Tosato-Rigo, I. Lacoue-Labarthe).

Qui écrit? En Provence ce sont femmes bien nées, filles et femmes de marchands, de patriciens, filles de la noblesse aussi, comme à Florence un peu plus tôt (C. Klapisch-Zuber) et en Suisse un peu plus tard (D. Tosato-Rigo et N. Hanafi). N'oublions jamais le contexte : ces femmes ont échappé à l'interdit d'écriture qui frappe nombre d'entre elles à la fin du Moyen Âge et tout au long de l'époque moderne. Pas de paysannes écrivaines, souligne Anna Iuso, à moins de les surprendre à broder leur existence sur un «grand drap de dessous ", telle Clélia Marchi de la province de Mantoue, dans un $\mathrm{XX}^{\mathrm{e}}$ siècle bien tardif. Au passage, relevons tout l'intérêt ici d'embrasser les différents supports d'écriture, du linge brodé à la consultation épistolaire (N. Hanafi), du tissu au papier.

Confrontées à la prise de plume - ou d'aiguille -, les femmes ont bien des points communs, et notamment une certaine appréhension et modestie même si, au fil du temps, elles semblent soumettre plus facilement leur corps à cette discipline et souhaitent en faire un « innocent délassement» (A. Iuso). Dans son étude sur les épistolières florentines, Christiane Klapisch-Zuber souligne à quel point elles

33 Base de données du GdR n²649 : www.ecritsduforprive.fr, voir détails note 9. 
"peinent à surmonter leur timidité et leur maladresse à l'écritoire ». De l'écriture déléguée à l'écriture quotidienne, sans fatigue ni souffrance, l'apprivoisement est long.

Écrire au quotidien consiste à noter les dépenses domestiques, s'occuper des affaires du ménage, de l'éducation des enfants, des choses banales de la maisonnée. L'écriture domestique est englobante et collective; elle a même souvent, dans le cadre de l'écriture des adolescentes, valeur d'« instrument de socialisation», comme le note Danièle Tosato-Rigo à propos d'Angletine Charrière de Sévery autour de 1800. Ces écrits sont rarement le lieu de l'épanchement, et même, $a$ priori, celui d'une quelconque expression de soi. Et pourtant, le moi féminin sourd à chaque ligne, celles d'un carnet que l'on fait volontiers sien - «ce livre contient l'estat de mes affaires », note Laure de Cambis (I. Luciani) -, et auquel on confie ses affections ou ses détestations, telle la florentine Monna Dora qui signe rageusement «Dora, ton ennemie ", devenant alors actrice à part entière de sa propre histoire. Le pacte de vérité souscrit ici, est un référent d'écriture que l'on retrouve jusque sous l'aiguille de la paysanne Clélia, "Gnanca na busia», pas même un mensonge.

Lorsqu'elles prennent la plume, les femmes sont «maitres d'ouvrage » (C. Klapisch-Zuber) ; de fait, elles deviennent scriptrices le plus souvent en l'absence de leur mari. Isabelle Luciani évoque le « royaume féminin» qu'elles bâtissent dans l'écriture, l'objet-livre matérialisant l'appropriation par les femmes de leur existence. Nombre d'auteures de ce volume soulignent à quel point celles-ci «s'emparent» de supports qui soutiennent un discours autonome (N. Hanafi, I. Lacoue-Labarthe). Elles acquièrent ainsi une véritable capacité d'agir sur le monde, de faire et d'imposer leurs choix; l'écriture est à la fois le miroir et le moyen de cette «agency » ${ }^{34}$. Une telle approche renouvelle notre regard sur la position des femmes au sein de la famille et plus largement des groupes sociaux d'appartenance. Toutes les auteures notent ici à quel point celle qui écrit est non seulement dans l'action, mais revendique en prime sa liberté de pensée et d'action.

34 Aujourd'hui traduit en français par «agentivité », ce concept est notamment utilisé par les philosophes, neuropsychologues et linguistes. Guilhaumou 2012. 
Cette écriture est pourtant souvent contrainte : comme bien des journaux de «demoiselles », le diaire d'Angletine jalonne le rôle social attendu d'une fille noble (D. Tosato-Rigo) ; nombre des mémoires de religieuses catalanes évoqués par Eulàlia Miralles et Verònica Zaragoza sont commandés par et pour l'institution et, tels les livres de comptes par exemple, ont vocation à servir la collectivité plutôt que leur auteure. Écrire au quotidien n'est donc pas toujours écrire sur soi, encore moins écrire librement.

La prise de parole que permet l'écriture quotidienne n'en reste pas moins un geste d'appréhension du monde et, au-delà, un acte d'affirmation, voire de construction, de découverte et d'appropriation de soi. Dora n'a certes pas son mot à dire au sujet du mariage de sa fille, du moins exprime-t-elle, et avec quelle vigueur, les sentiments que lui inspire sa soumission forcée (C. Klapisch-Zuber) ; écrire est encore, pour les sionistes arrivées en Palestine au début du XX $\mathrm{XX}^{\mathrm{e}}$ siècle, se dresser contre un sort auquel elles ne se résignent pas (I. Lacoue-Labarthe).

Si le geste quotidien de s'asseoir, plume en main, et de garder un moment pour soi construit la routine des jours, il devient la " passion dévorante » d'Amélie Weiler (A. Iuso), tandis que le journal d'Émilie Serpin, de "passe-temps le plus doux » se voit promu rival de Dieu dans les préoccupations de la pieuse jeune femme (P. Lejeune).

L'écriture quotidienne finit parfois par cannibaliser l'existence de ces femmes, arrimées à ce précieux sésame, qui leur ouvre, hors de la sphère domestique et par-delà l'égotisme trop souvent imputé aux écrits de soi, l'accès à d'autres horizons. D'abord destinées à des proches, les correspondances tissent des sociabilités parfois très élargies et insèrent les épistolières dans des réseaux européens non dénués de résonance politique (M.-C. Hoock-Demarle); elles brouillent la distinction entre sphère privée et espace public (M. Caffiero). Entamée par nécessité ou par désœuvrement, l'écriture journalière nourrit des appétits inattendus - la jeune Émilie Serpin se rêve en poétesse -, alimentée par la confiance gagnée à chaque mot, au point de devenir l'antichambre d'une œuvre plus ambitieuse, voire l'œuvre d'une vie (les Briefromane de Bettina von Arnim en sont un exemple, présenté par M.-C. Hoock-Demarle).

Ouverture pour leurs auteures, ces écrits longtemps négligés constituent aussi pour les historien-ne-s de nouvelles voies de réflexion 
sur le passé en interrogeant les connaissances historiques jusqu'alors admises (M. Caffiero, I. Lacoue-Labarthe) et en restituant à la moitié longtemps invisible de l'humanité sa place de sujet ou, mieux encore si possible, d'actrice autonome douée de raison et d'émotion.

\section{Bibliographie}

ALBERT Jean-Pierre, 1993, «Écritures domestiques », in Daniel FABRE (dir.), Écritures ordinaires, Paris, POL Éditeur, p. 37-95.

Baggerman Ariane, 2002, «Autobiography and Family Memory in the Nineteenth Century ", in Rudolf DEKKER (ed.), Egodocuments and History. Autobiographical Writing in its Social Context since the Middle Ages, Hilversum, Verloren, Reeks Publikaties van de Faculteit der Historische en Kunstwetenschappen 38, p. 161-175.

BARDET Jean-Pierre, ARnoul Elisabeth \& François-Joseph RugGiU (dir.), 2010, Les écrits du for privé en Europe du Moyen Âge à l'époque contemporaine: enquêtes, analyses, publications, Bordeaux, Presses universitaires de Bordeaux.

BARDET Jean-Pierre \& François-Joseph RugGIU (dir.), 2005, Au plus près du secret des cours? Nouvelles lectures historiques des écrits du for privé en Europe du XVIe au XVIII siècle, Paris, Presses de l'Université Paris-Sorbonne.

BARTHÉLÉMY Pascale, 2009, «Je suis une Africaine... j’ai vingt ans. Écrits féminins et modernité en Afrique occidentale française (c. 1940-c. 1950)», Annales, Histoire, Sciences sociales, 64/4, p. 825-852.

BEAUVOIR Simone de, 1976 [1949], Le deuxième sexe, vol. I-II, Paris, Gallimard.

BODJ-POuYE Aïssatou \& Éloi FICQUET, 2009, «Cultures de l'écrit en Afrique. Anciens débats, nouveaux objets ", Annales, Histoire, Sciences sociales, 64/4, p. 751-764

Broomhall Susan \& Colette WINN, 2008, Les femmes et l'bistoire familiale (XVIe-XVII siècle), Paris, Champion.

CAFFiero Marina, 2008, Rubare le anime. Diario di Anna del Monte ebrea romana, Rome, éd. Viella, coll. «La memoria restituita ».

Caffiero Marina \& Manola Ida Venzo (dir.), 2007, Scritture di donne, Rome, éd. Viella, coll. «La memoria restituita ».

CASSAN Michel, BARDET Jean-Pierre \& François-Joseph RUGGIU (dir.), 2007, Les écrits du for privé : objets matériels, objets édités, Limoges, Presses universitaires de Limoges.

Castillo Gómez Antonio, 2007, El legado de Mnemosyne. Las escrituras del yo a través del tiempo, Gijón, Trea.

CASTILlo GÓmez Antonio (dir.), Sierra Blas Verónica (ed.), 2007, Escrituras del yo. Del libro de cuentas al diario intimo (Edades Media, Moderna y Contemporánea), Gijón, Trea.

CAZAlé Bérard Claude, 2009, Donne tra memoria e scrittura. Fuller, Weil, Sachs, Morante, Rome, Carocci. 
CaZAlé-BÉrard Claude \& Christiane Klapisch-Zuber, 2004, « Mémoires de soi et des autres dans les livres de famille italiens », Annales HSS, 4, juillet-août, p. 805-826.

CHARTIER Roger (dir.), 1996, Les pratiques de l'écriture ordinaire dans les sociétés de l'Ancien Régime, Lyon, Université Louis Lumière-Lyon II, GRS.

DAVIS Natalie Zemon, 1995, Juive, catholique, protestante. Trois femmes en marge au XVII siècle, Paris, Seuil.

DEKKER Rudolf, 1995, «Les egodocuments aux Pays-Bas du XVIe au XVIII" siècle », Bulletin du bibliophile, p. 317-333.

DEKKER Rudolf (ed.), 2002, Egodocuments and History, Autobiographical Writing in its Social Context since the Middle Ages, Hilversum, Verloren.

Del Val González De La Peña Maria (dir.), 2005, Mujer y cultura escrita, del mito al siglo XXI, Gijon, Ediciones TREA.

DesLandres Dominique, 2011, «Se dire, s’écrire, se faire: pour une histoire de l'agentivité féminine et de sa représentation dans l'espace français au XVII siècle ", in Jean-Pierre BARDET \& François-Joseph RUGGIU (dir.), Les usages de l'écrit du for privé (Afriques, Amériques, Asies, Occidents, Orients), sous presse.

Diaz Brigitte \& Jürgen SIESS (dir.), 2006, L'épistolaire au féminin. Correspondances de femmes, ХVIII $-X X^{e}$ siècles, Caen, Presses Universitaires de Caen.

DuCHÊNE Roger, 1970, Réalité vécue et art épistolaire. Madame de Sévigné et la lettre d'amour, Paris, Bordas.

—, 2006, Comme une lettre à la poste. Le progrès de l'écriture personnelle sous Louis XIV, Paris, Fayard.

FABRE Daniel (dir.), 1993, Écritures ordinaires, Paris, POL Éditeur.

—, 1997, Par écrit. Ethnologie des écritures quotidiennes, Paris, Éditions de la MSH.

—, 2000, «Christine PlantÉ (dir.), L'épistolaire, un genre féminin ?, Paris, Champion, 1998 », Clio. Histoire, femmes et sociétés, 11, p. 218-220.

FABRE Daniel \& Agnès FINE (dir.), 2000, " Parler, chanter, lire, écrire », Clio, Histoire, Femmes et Sociétés, 11.

FINE Agnès, 2000, "Écritures féminines et rites de passage », Communications, 70, p. 121-142.

FInE Agnès, LABro Stéphanie \& Claire LORQUIN, 1993, «Lettres de naissance », in Daniel FABRE (dir.), Écritures ordinaires, Paris, POL Éditeur, p. 116-147.

FoIsIL Madeleine, 1986, «L'écriture du for privé », in Philippe ARIÈs, Georges DuBY, \& Roger CHARTIER (dir.), Histoire de la vie privée, vol. 3, De la Renaissance aux Lumières, Paris, Seuil, p. 331-369.

Guilhaumou Jacques, 2012, «Autour du concept d'agentivité », Rives Méditerranéennes, 41, p. 25-34. 
Hammerle Christa \& Li Gerhalter (hrsg.), 2010, Apokalyptische Jabre. Die Tagebücher der Therese Lindenberg (1938 bis 1946), L'HOMME Archiv, Band 2, Köln/Weimar/Wien, Böhlau.

Histoire et Archives de soi, 2002, Sociétés et Représentations, CREDHESS, $\mathrm{n}^{\circ} 13$.

Hoock-Demarle Marie-Claire, 1990, La rage d'écrire. Femmes écrivains en Allemagne, de 1790 à 1815, Aix-en-Provence, Alinéa.

—, 2008, L'Europe des lettres. Réseaux épistolaires et construction de l'espace européen, Paris, Albin Michel.

Houbre Gabrielle, 1996, «Les jeunes filles au fil du temps », Clio. Histoire, Femmes et Sociétés, 4, URL : http://clio.revues.org/428 ; DOI : 10.4000/clio.428

Iuso Anna, 1997, «Les archives du moi ou la passion autobiographique », Terrain, 28, p. $125-138$.

Iuso Anna (dir.), 1998, Scritture di donne. Uno sguardo europeo, Sienne, Protagon Editori Toscani.

—, (dir.), 2011, La face cachée de l'autobiographie, Carcassonne, Garae Hésiode.

Kisseliova Evguenia, 2000, Une femme russe dans le siècle. Journal d'Evguenia Kisseliova. 1916-1991, Paris, Albin Michel.

KLAPISCH-Zuber Christiane, 1990, La maison et le nom. Stratégies et rituels dans l'Italie de la Renaissance, Paris, EHESS.

—, 2001, «Les vies de femmes des livres de famille florentins », Mélanges de l'École française de Rome, Italie et Méditerranée, 113/1, p. 107-121.

LACOUE-LABARTHE Isabelle, 2011, «Lettres et journaux de femmes. Entre écriture contrainte et affirmation de soi », Tumultes, 36, p. 113-132.

LAHIRE Bernard, 1993, La raison des plus faibles. Rapport an travail, écritures domestiques et lectures en milieux populaires, Lille, Presses universitaires de Lille.

—, 1995, «Écritures domestiques : la domestication du domestique », Informations sur les sciences sociales, 34/4, p. 567-592.

—, 1997, «Masculin-Féminin. L'écriture domestique », in FABRE Daniel (dir.), Par écrit. Ethnologie des écritures quotidiennes, Paris, Éditions de la Maison des Sciences de l'Homme, p. 145-164.

LANZ Anne-Marie, 2009, Dans le fleuve de l'oubli : journal de Catherine Charrière de Sévery, Maryland, UMI Research Press.

LE MAO Caroline, 2006, «Une redoutable femme d'affaires : la première présidente Olive de Lestonnac (1572-1652) ", Annales du Midi, t. 118, n²53, p. 11-29.

Lejeune Philippe, 1993a, Lucile Desmoulins, Journal 1788-1793, Paris, Éditions des Cendres.

—, 1993b, Le moi des demoiselles, Paris, Seuil.

—, 2005, Signes de vie. Le pacte autobiographique, 2, Paris, Seuil. 
Lejeune Philippe \& Catherine BOGAERT, 2003, Un journal à soi, histoire d'une pratique, Paris, Éditions Textuel.

LemaitRe Nicole, 2006, «Les livres de raison en France (fin XIII ${ }^{\mathrm{e}-X I X^{\mathrm{e}}}$ siècle) », Testo \& Senso, 7, 2006, p. 1-18, disponible sur le site www.testoesenso.it.

Lemaitre Nicole \& Sylvie Mouysset (dir.), 2010, Entre mémoire et histoire, écriture ordinaire et émergence de l'individu, Paris, éditions du CTHS. Édition électronique: http://cths.fr/ed/edition.php?id=5327.

MANDROU Robert, 1961, Introduction à la France moderne, essai de psychologie historique (1500-1640), Paris, Albin Michel. Nombreuses rééditions, notamment 1998.

MOUYSSET Sylvie, 2005, « Male or Female? Gender and the Family Record in Early Modern France", 33e colloque annuel de la Western Society for French History, Colorado Springs (USA). Édition électronique: www.wsfh.org.

—, 2007, Papiers de Famille. Introduction à l'étude des écrits du for privé (France, XVe-XIXe siècle), Rennes, Presses universitaires de Rennes.

—, 2009, «De mémoire, d'action et d'amour : les relations hommes/femmes dans les écrits du for privé français au XVII' siècle », Dix-septième siècle, 3, n²44, p. 393-408.

Mouysset Sylvie, BARdet Jean-Pierre \& François-Joseph Ruggiu (dir.), 2010, Car c'est moy que je peins. Écriture de soi, individu et liens sociaux (Europe, XV $\mathrm{V}^{e} \mathrm{XX} \mathrm{X}^{e}$ siècle), Toulouse, Méridiennes.

Perrot Michelle, 1979, Les Filles de Karl Marx. Lettres inédites, Introduction, Paris, Albin Michel, p. 9-50. Lettres traduites et présentées par Olga MEIER et Michel TreBITSCH.

—, 1986, «Journaux intimes. Jeunes filles au miroir de l'âme», Adolescence, IV/1, p. 29-36.

—, 1987, « Pratiques de la mémoire féminine », Traverses, 40, IV, p. 19-29.

Perrot Michelle \& Georges RibeiLl, 1985, Journal intime de Caroline B., Paris, Montalba.

Planté Christine, 1992, «Une lettre de femme. La Lettre et le poème chez Desbordes-Valmore », Mélanges offerts à Georges Lubin, Brest, CCJI-CNRS, p. 203-214

—, 1994, «L'Intime comme valeur publique. La correspondance d’Eugénie de Guérin ", in Mireille Bossis (dir.), La Lettre à la croisée de l'individuel et du social, Paris, Kimé, p. 82-90.

—, 1998a, L'Épistolaire, un genre féminin ?, Paris, Honoré Champion.

—,1998b, «Une lettre d'homme, une lettre de femme. Traitement sexué de l'épistolaire dans Le Marquis de Villemer de George Sand ", in Christine Planté (dir.), L'épistolaire, un genre féminin ?, Paris, Honoré Champion.

POUTRIN Isabelle, 1995, Le voile et la plume: autobiographie et sainteté féminine dans l'Espagne moderne, Madrid, Casa de Velázquez.

—, 2005, «L'identité sociale dans les écrits autobiographiques féminins de l’Espagne moderne », in Jean-Pierre BARDET \& François-Joseph RUGGIU (dir.), Au plus près $d u$ secret des cours? Nouvelles lectures historiques des écrits du for privé en Europe du XVIe au ХИII' siècle, Paris, Presses de l’Université Paris-Sorbonne, p. 93-110. 
Rachmanova Alia, 2010, Une crémière russe à Vienne. Journal d'une émigrée (1925-1927), Paris, Payot, coll. « Petite Bibliothèque Payot », trad. Chantal Le Brun Kéris.

RIVES Danielle, 2012, «Le crépuscule d'une communauté religieuse au miroir de son livre de raison : la Visitation d'Albi de 1783 à 1791 », à paraittre dans la revue catalane Manuscrits.

SiBLOT Yasmine, 2006, " "Je suis la secrétaire de la famille !" La prise en charge féminine des tâches administratives entre subordination et ressource ", Genèses, 64, p. 46-66.

SimONET-TENANT Françoise, 1990, Le journal intime, genre littéraire et écriture ordinaire, Paris, Hatier.

—, 2001, «Catherine Pozzi : Le Journal comme laboratoire de l'œuvre », Genesis, 16, «Autobiographies », p. 75-95.

Stistrup JeNSEN Merete, 2000, «La notion de nature dans les théories de l'écriture féminine », Clio, Histoire, Femmes et Sociétés, 11. http://clio.revues.org/index218.html

Timmermans Linda, 1993, L'accès des femmes à la culture (1598-1715), Paris, Honoré Champion.

Tosato-Rigo Danièle, 2010, «Pratiques de l'écrit et histoire par la marge. Autour des "egodocuments" en Suisse romande au XVIII" siècle", Revue suisse d'Art et d'Archéologie, 67/4, p. 259-268.

VERCIANI Laura, 2001, Le Moi et ses diables, autobiographie spirituelle et récit de possession au XVII siècle, traduit de l'italien par Arlette Estève, Paris, Honoré Champion.

VINYOLES VIDAL Térésa, 2005, « La cotidianidad escrita por una mujer del siglo XV », in Del Val GonzÁlez de la PeÑa Maria (dir.), Mujer y cultura escrita, del mito al siglo XXI, Gijón, Trea.

VIOLLET Catherine, 2006, «Aux frontières de la correspondance : journaux féminins de l'aristocratie russe (début du XIX"e siècle) », Épistolaire, 32, p. 71-79. 